\title{
Memoquin: A Multi-Target-Directed Ligand as an Innovative Therapeutic Opportunity for Alzheimer's Disease
}

\author{
Maria Laura Bolognesi,* Andrea Cavalli,*广 and Carlo Melchiorre* \\ *Department of Pharmaceutical Sciences, Alma Mater Studiorum, University of Bologna, 40126 Bologna, Italy, and ${ }^{\dagger}$ Department \\ of Drug Discovery and Development, Italian Institute of Technology, 16163 Genova, Italy
}

\begin{abstract}
Summary: Alzheimer's disease is currently thought to be a complex, multifactorial syndrome, unlikely to arise from a single causal factor; instead, a number of related biological alterations are thought to contribute to its pathogenesis. This may explain why the currently available drugs, developed according to the classic drug discovery paradigm of "one-molecule-one-target," have turned out to be palliative. In light of this, drug combinations that can act at different levels of the neurotoxic cascade offer new avenues toward curing Alzheimer's and other neurodegenerative diseases. In parallel, a new strategy is emerging - that of developing a single chemical
\end{abstract}

entity able to modulate multiple targets simultaneously. This has led to a new paradigm in medicinal chemistry, the "multitarget-directed ligand" design strategy, which has already been successfully exploited at both academic and industrial levels. As a case study, we report here on memoquin, a new molecule developed following this strategy. The in vitro and in vivo biological profile of memoquin demonstrates the suitability of the new strategy for obtaining innovative drug candidates for the treatment of neurodegenerative diseases. Key Words: Multifunctional compounds, AD11 mice, acetylcholinesterase, amyloid, antioxidant, benzoquinones, tau hyperphosphorylation.

\section{INTRODUCTION}

Alzheimer's disease (AD) is the most common neurodegenerative disease among the elderly. It affects 26 million people worldwide, ${ }^{1}$ and its prevalence is increasing so greatly that the number of individuals with $\mathrm{AD}$ is expected to more than triple between the present and 2040. Despite the huge proportions of the phenomenon, ${ }^{2}$ $\mathrm{AD}$ remains incurable and fatal, and this fact represents an urgent need and unmet goal for the research community.

Until 2003, the therapeutic options available for patients with $\mathrm{AD}$ were limited to one class of drugs, the inhibitors of acetylcholinesterase (AChE), which offer only symptomatic relief. Their action is restricted to the amplification of residual cholinergic activity by slowing degradation of acetylcholine (ACh) after its release at the synapses. ${ }^{3}$ Since 2003 , a newer drug, memantine, has

Address correspondence and reprint requests to: Maria Laura Bolognesi, Ph.D., Department of Pharmaceutical Sciences, Alma Mater Studiorum, University of Bologna, Via Belmeloro 6, 40126 Bologna, Italy. E-mail: marialaura.bolognesi@unibo.it. Or Andrea Cavalli, Ph.D., Department of Pharmaceutical Sciences, Alma Mater Studiorum, University of Bologna, Via Belmeloro 6, 40126 Bologna, Italy. E-mail: andrea.cavalli@unibo.it. been approved. Its mechanism of action relies on the prevention of overstimulation of the N-methyl-D-aspartate (NMDA) receptors, which probably contribute to the pathogenesis of $\mathrm{AD}$ by causing excitotoxicity. To date, however, no treatment has been unequivocally proven to alter or prevent disease progression, and many agents entered in clinical trials have proven ineffective. ${ }^{4}$

As a consequence, drug discovery research has been driven-medically, commercially, and intellectually—by the need for therapeutics with disease-modifying effects that can get to the root of the disease. This need has often been equated with the identification and exploitation of novel targets, but these efforts have often proved disappointing.

An exemplary case is amyloid- $\beta(\mathrm{A} \beta)$. Over the last 30 years, a plethora of targets has been suggested in the attempt to identify the causative factors of neurodegeneration. Among these, a great deal of evidence from a variety of genetic, pathological, and biochemical studies suggested that $A \beta$ played a pivotal causal role in the pathogenesis of AD. According to the "amyloid hypothesis," one of the major neuropathological hallmarks of $\mathrm{AD}$ is the altered production, aggregation, and deposition of $\mathrm{A} \beta$, which results in amyloid plaque formation. ${ }^{5}$ Therefore, much effort was directed at developing drugs 
to inhibit the production, aggregation, or neurotoxicity of $\mathrm{A} \beta .^{6}$ More recently, the scientific community realized that the pathogenetic scenario might be even more complex. Abnormal protein assemblies appear to initiate a cascade of interrelated pathological events, ultimately leading to neuronal death. Answers to more specific questions have yielded other targets, ${ }^{6}$ which have entered pharmaceutical company pipelines. However, it has been difficult to determine which of these pathways is the most important in the onset of $\mathrm{AD}$, and which should be targeted for the development of new therapeutic agents. ${ }^{7}$ Therefore, although the speed of research has increased in recent years, $\mathrm{AD}$ remains an area of exceptional clinical need.

In the multifactorial nature of the disease, scientists have identified the reasons for these pitfalls. ${ }^{8}$ Experimental evidence points to the involvement of several targets and pathways in the AD pathogenesis (FIG. 1), but all drugs developed to date are monofunctional, hitting only a single target among the many involved. Therefore, these drugs are inherently insufficient for the treatment of complex diseases like $\mathrm{AD}$, which have multiple pathogenic factors. ${ }^{9}$ Different pharmacological approaches offer possible ways of overcoming the problems arising from the use of monofunctional drugs. ${ }^{10}$ First, a combination of drugs, which offers the prospect of additional benefits, can be effectively used to target the multiple pathological processes. Combination therapy has already proven successful in the treatment of similarly complex diseases, such as cancer, HIV, and hypertension, where the technique achieves maximum efficacy by attacking several targets simultaneously, exploiting synergy, and minimizing individual toxicity. In AD as well, combination therapy is becoming the standard of care. ${ }^{11}$ Usually, a therapeutic regimen comprises the concomitant use of one of the marketed AChE inhibitors (AChEIs) and memantine, due to their distinct mechanisms of action. Moreover, co-administration of other traditional and nontraditional therapies (vitamin E, ginkgo biloba) may form a successful strategy in the near future. ${ }^{12}$ The safety and effectiveness of these and other agents for AD patients are still matters of debate. However, there is an increasing body of evidence that suggests that synergic effects may arise from combining agents that already have some proven efficacy in AD therapy and that are directed at different mechanisms in the pathogenic chain.

It is critical to recognize, however, that combining two or more drugs always raises the potential for greater side effects. These may include the known side effects of each drug, or completely unexpected side effects caused by interactions between the respective drugs. ${ }^{13}$ Another therapeutic option is now emerging, based on the paradigm that a rationally designed, single molecule may possess multiple concomitant biological properties. Clearly, therapy with such a single multimodal drug would have inherent advantages over combination therapy. ${ }^{14}$ Such therapy would prevent the challenge of administering multiple single-drug entities that could have different bioavailability, pharmacokinetics, and metabolism. Furthermore, in terms of pharmacokinetic and toxicological optimization, the clinical development of a drug able to hit multiple targets should not, in principle, be different from the development of any other single lead molecule. This therapy thus offers a far more simple approach than combination therapy. In addition, the risk of possible drug-drug interactions would be reduced and the therapeutic regimen greatly simplified, with the prospect of enhanced patient compliance, which is a critical issue in $\mathrm{AD}$ care. ${ }^{15}$

The development of an effective multimodal treatment is, however, not so clear cut. Neurodegeneration in AD is the result of a several-step process, and addressing with a single drug the molecular dynamics of disease progression is not an easy task. When designing a new chemical entity, one should keep in mind that AD has many stages of progression, and it is crucial to assess the relationship between the progression timeline and a specific molecular target. ${ }^{16}$

\section{MEMOQUIN DESIGN STRATEGY}

In 1998, aware that innovative approaches to drug design were required to obtain real disease-modifying drug candidates, our group embarked on a new medicinal chemistry project to discover molecules endowed with a multifunctional profile against $\mathrm{AD}$. At that time, our starting point was a solid background in the cholinergic system and an arsenal of lead compounds endowed with the ability to modulate different cholinergic targets. In particular, the polyamineamide caproctamine $(\mathbf{1})^{17}$ was of particular interest as a lead candidate, due to a well balanced affinity profile as an AChEI and a competitive muscarinic $\mathrm{M}_{2}$ receptor antagonist (FIG. 2). The idea had been advanced that drugs that antagonize selectively presynaptic muscarinic $\mathrm{M}_{2}$ autoreceptors might also be useful in $\mathrm{AD}$, as they would facilitate $\mathrm{ACh}$ release. ${ }^{18}$ Therefore, we reasoned that 1 may well be capable of restoring cholinergic activity in the brain affected by AD pathology by decreasing ACh hydrolysis rates and at the same time increasing ACh release in the synapse. Moreover, it displays a dimeric flexible structure, which allows it to contact both active and peripheral sites of AChE, ${ }^{19}$ as hypothesized by properly addressed molecular modeling studies. ${ }^{17}$ This behavior has important consequences for the therapeutic potential of $\mathbf{1}$ against $\mathrm{AD}$, in light of the discovery that AChE plays a key role during an early step in the development of senile plaques, acting as a pathological molecular chaperone. ${ }^{20}$ It was postulated that $\mathrm{AChE}$ binds to the $\mathrm{A} \beta$ non-amyloidogenic form, inducing a conformational transition to the amyloido- 


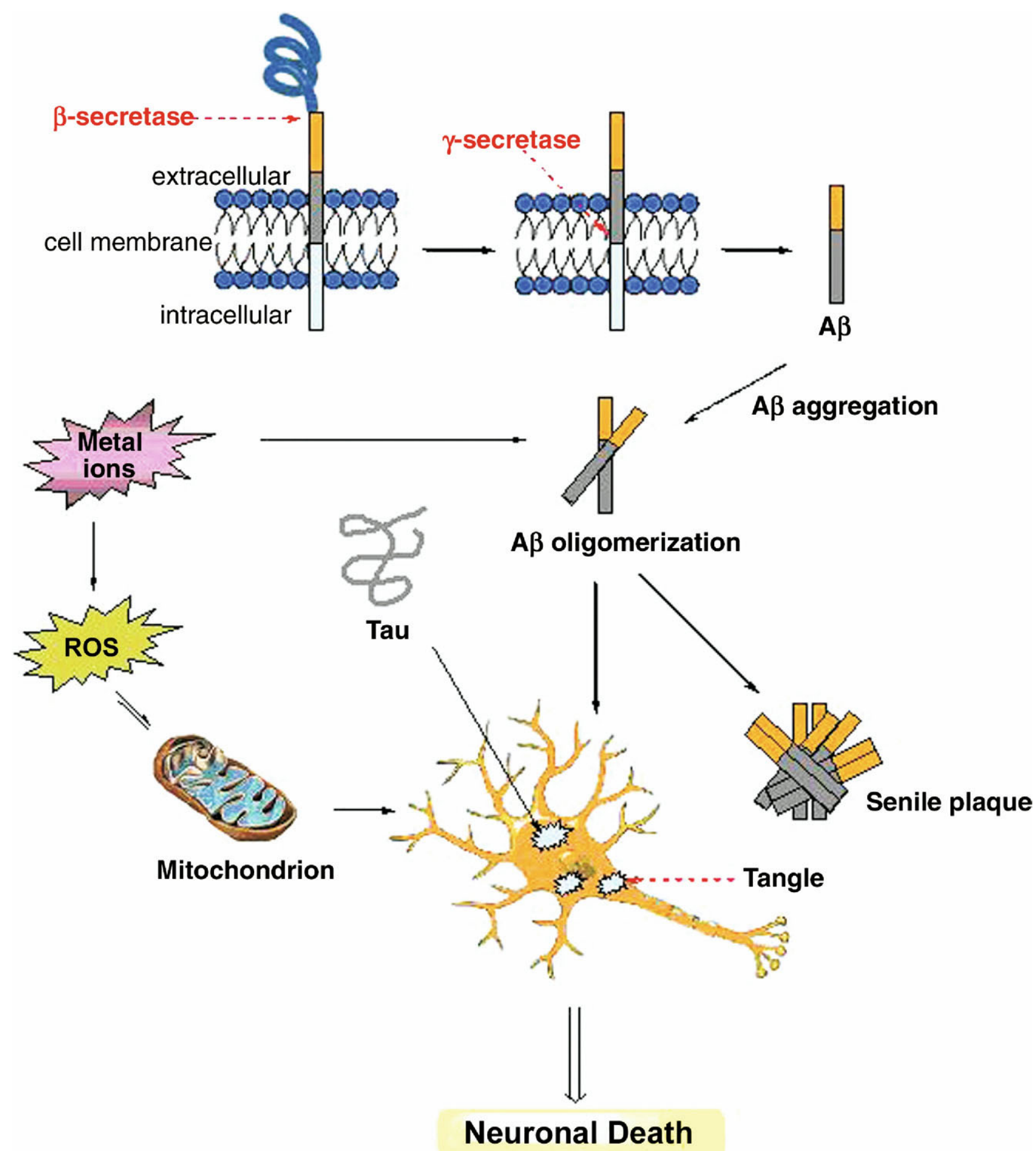

FIG. 1. Schematic illustration of the multiple pathways that have been recognized as fundamental in the pathogenesis of $A D$. A $\beta=$ amyloid- $\beta$; ROS $=$ reactive oxygen species; tau $=$ the hyperphosphorylated form of the tau protein.

genic conformation with subsequent amyloid fibril aggregation. ${ }^{21}$ In fact, $\mathrm{AChE}$ directly promotes in vitro the assembly of $\mathrm{A} \beta$ peptide into amyloid fibrils, forming stable AChE-A $\beta$ complexes. ${ }^{22}$ Structural analysis by Xray crystallography ${ }^{23}$ and competition assays with AChEIs $^{22}$ clearly identified enzyme peripheral anionic site (PAS) as a locus of protein interaction with $\mathrm{A} \beta$. All these results have constituted the premise for medicinal chemists to design a new class of dual-binding site inhibitors thatable to simultaneously interact with the two sites - might alleviate the cognitive deficit in $\mathrm{AD}$ and, more importantly, address the etiology of the disease. ${ }^{24-26}$

In view of early evidence that polyamineamides were versatile molecular scaffolds suitable for designing com- 


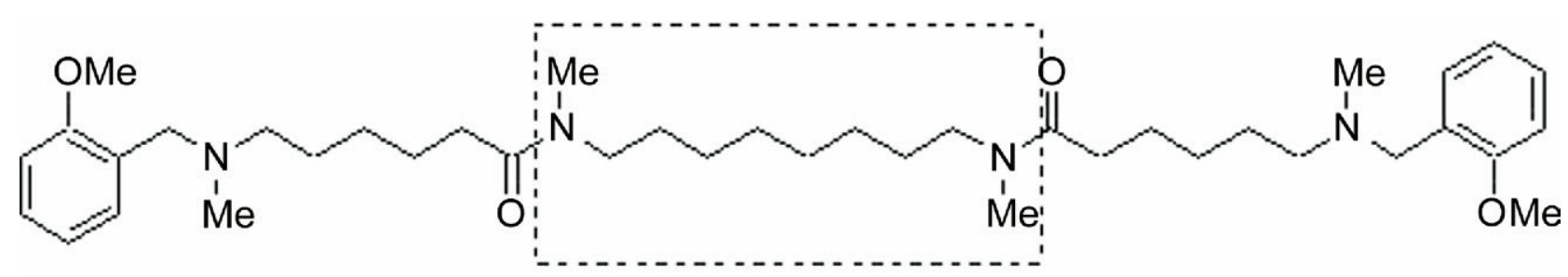

1: caproctamine

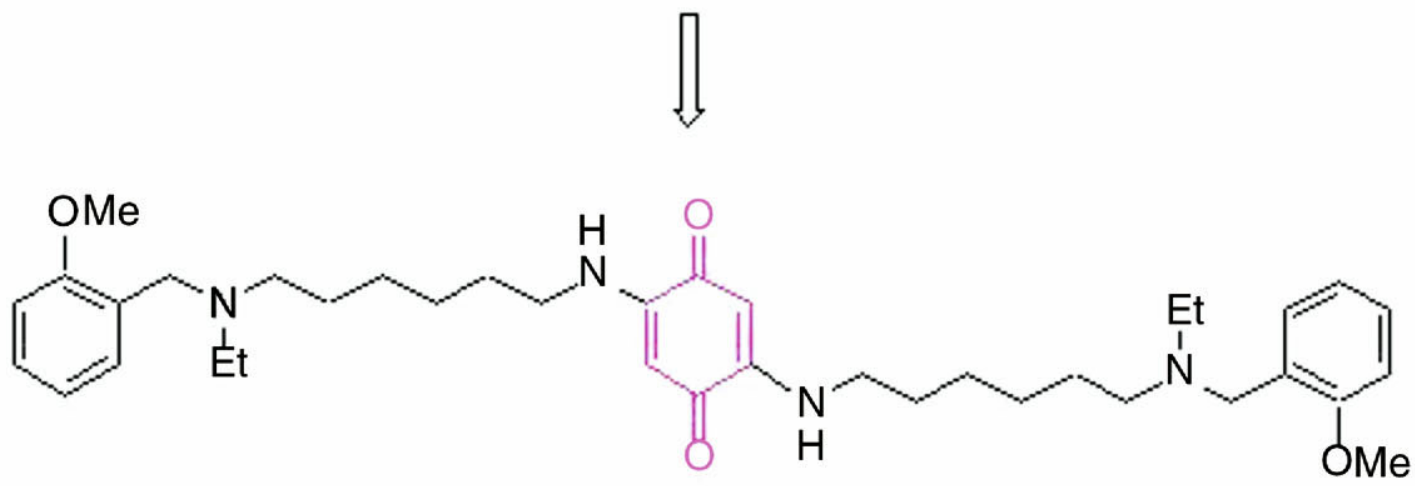

\section{2: memoquin}

FIG. 2. Design strategy leading to the MTDL 2.

pounds able to recognize multiple protein targets, ${ }^{27}$ we considered the possibility of modifying the structure of $\mathbf{1}$ to obtain a multi-target-directed ligand (MTDL) ${ }^{28}$ In addition to the cholinergic properties, we aimed to add to caproctamine another activity beneficial for $\mathrm{AD}$, such as the antioxidant property. Our interest was prompted by the observation that the synthetic derivative of coenzyme $\mathrm{Q}(\mathrm{CoQ})$, idebenone, had been shown in a clinical trial to improve cognitive function and behavioral deficits in patients with mild to moderate $\mathrm{AD} .{ }^{29}$ Moreover, treatment with idebenone, a potent mitochondrial antioxidant, protected the hippocampus neurons against $\mathrm{A} \beta(1-40)$ induced neurotoxicity. ${ }^{30}$

Currently, extensive literature points to oxidative stress as pivotal in the pathogenesis of $\mathrm{AD}$, suggesting that it occurs prior to the onset of symptoms in $\mathrm{AD}$, and that oxidative damage occurs before plaque formation. ${ }^{31}$ It is worth noting that low-dose idebenone is marketed in Italy (Mnesis [Takeda Italia Farmaceutici, Rome, Italy], Daruma [Wyeth Lederle, Aprilia, Italy]) for cognitive disorders.

Thus, because of their different mechanisms of action, combination therapy with idebenone and an AChEI emerged as a therapeutic option for the management of $\mathrm{AD}$ that is still useful today. ${ }^{32}$ We therefore envisioned the creation of a multi-target-directed new molecule by inserting the 1,4-benzoquinone radical scavenger moiety of $\mathrm{CoQ}$ and analogues into the polyamine backbone of $\mathbf{1}$. We decided to introduce the benzoquinone nucleus to replace the inner polymethylene chain. In the resulting 2,5-bis- $\{6-$ [ethyl-(2-methoxy-benzyl)-amino]-hexylamino\}-[1,4]benzoquinone (memoquin; 2), ${ }^{33}$ the two nitrogen atoms in positions 2 and 5 of the benzoquinone moiety are amide-like in character, precisely mimicking the amide bonds of $\mathbf{1}$ (FIG. 2). Due to a resonance effect, a hydrophobic and planar $\pi$ system is generated, which has been recognized as an important design feature for obtaining high-binding specificity with $A \beta$ and for perturbing protein-protein interactions in the fibrillogenesis process. ${ }^{34}$

A straightforward synthetic strategy was developed to provide both a high-yield and large-scale amount of the molecule. $^{35}$

To disclose the multiple mechanisms of action of this novel MTDL, a number of different in vitro and in vivo assays were performed, accounting for AChE inhibition, $\mathrm{A} \beta$ aggregation, antioxidant effects, and BACE-1 inhibition. ${ }^{33,36,37}$

\section{IN VITRO PROFILE}

In FIG. 3 and Table 1, we report a summary of all the in vitro activities of memoquin.

\section{Antioxidant activity of memoquin}

The antioxidant properties of $\mathbf{2}$ were verified by testing its ability to neutralize free radicals. The molecule was able to decrease the formation of free radicals with a $44.1 \pm 3.7$ percentage of inhibition. The antioxidant capacity appeared to be slightly lower than that shown by a standard reference compound, such as trolox $(57.6 \% \pm$ 


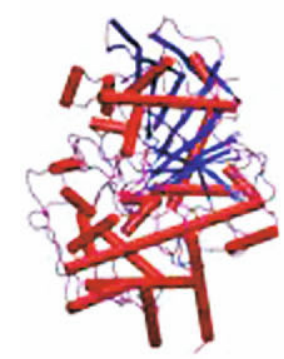

AChE

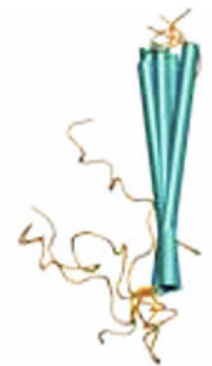

$A \beta(1-42)$ (self)

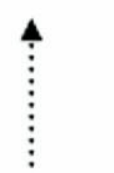

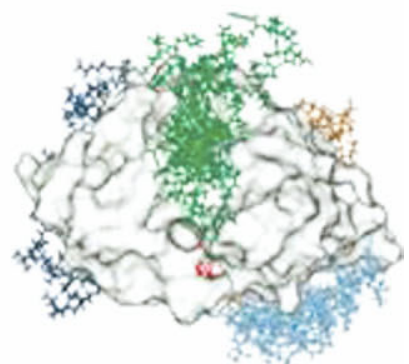

$A \beta(1-40)$

(AChE)<smiles>CI</smiles><smiles>CCN(CCCCCCI)Cc1ccccc1OC</smiles><smiles>C=NC1=CC(=O)C(NCCCCCCN(CC)Cc2ccccc2OC)=CC1=O</smiles>

\section{Memoquin (2)}
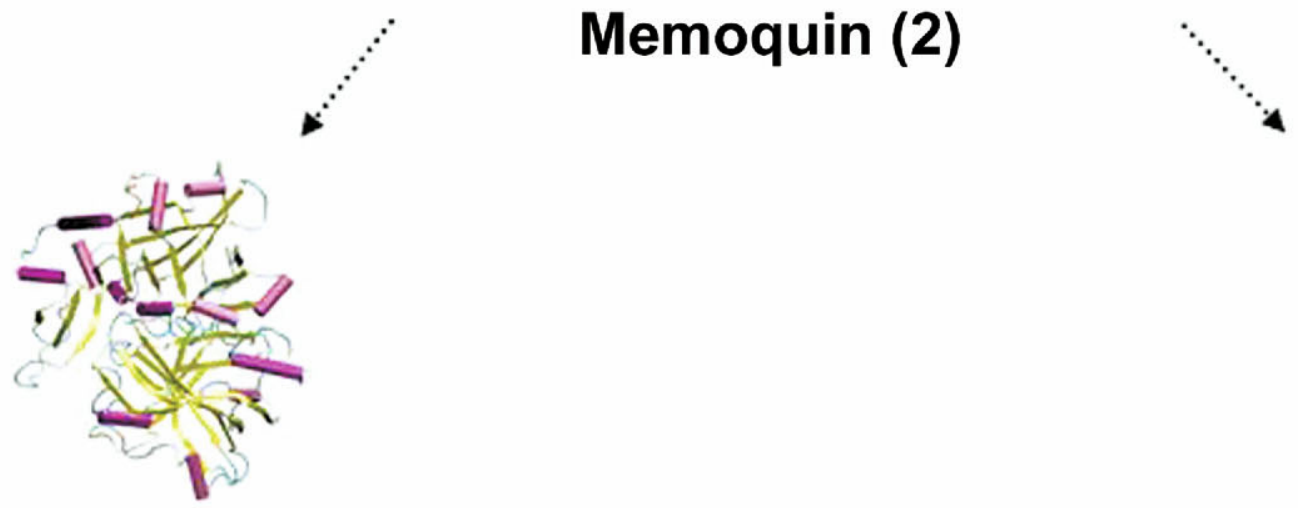

\section{BACE-1}

FIG. 3. Schematic representation of the in vitro MTDL profile of 2.

$0.9 \%$ ). However, it should be noted that in the experiment carried out, $\mathbf{2}$ was in the oxidized form, which, in vivo, would be readily transformed into the more antioxidant-reduced form. In fact, different studies on the antioxidant property of other 1,4-benzoquinone derivatives, such as CoQ and idebenone, have conclusively demonstrated that it pertains exclusively to their hydroquinone form, since the quinone, in principle, cannot scavenge radicals. ${ }^{38} \mathrm{NAD}(\mathrm{P}) \mathrm{H}$ :quinone oxidoreductase 1 (DT-diaphorase, NQO1) was shown to be responsible for the regeneration and maintenance of the CoQ-reduced state. This inducible enzyme catalyzes the two- electron reduction of quinones to hydroquinones, bypassing production of semiquinones and thus providing a shunt that competes with the formation of reactive oxygen species (ROS). More interestingly, NQO1 is increased in $\mathrm{AD}^{39}$ in response to the shift of redox balance typical of the pathology. ${ }^{40}$ Therefore, we might speculate that, being specifically reduced by NQO1 into the corresponding hydroquinone, $\mathbf{2}$ may exert its antioxidant activity specifically in those brain regions affected by $\mathrm{AD}$, where a co-localization of NQO1 activity with AD pathology has been experimentally verified. ${ }^{41}$

Therefore, $\mathbf{2}$ was tested with respect to its ability to 
Table 1. Activities of Memoquin and Reference Compounds at the Selected Molecular Targets

\begin{tabular}{lll}
\hline \multicolumn{1}{c}{ Target } & \multicolumn{1}{c}{2 (Memoquin) } & \multicolumn{1}{c}{ Reference Compound } \\
\hline NQO1 & $\mathrm{V}_{\max }=3480(\mu \mathrm{mol} / \mathrm{min}) / \mathrm{mg} ; \mathrm{K}_{\mathrm{M}}=$ & $\mathrm{V}_{\max }=7286(\mu \mathrm{mol} / \mathrm{min}) / \mathrm{mg} ; \mathrm{K}_{\mathrm{M}}=$ \\
& $12.7 \mu \mathrm{M}$ & $1.20 \mu \mathrm{M}^{*}$ \\
$\mathrm{hAChE}$ & $\mathrm{IC}_{50}=1.55 \pm 0.11 \mathrm{nM} ; \mathrm{K}_{\mathrm{i}}=2.60 \pm$ & $\mathrm{IC}_{50}=23.1 \pm 4.8 \mathrm{nM} ; \mathrm{K}_{\mathrm{i}}=20.5 \pm$ \\
& $0.48 \mathrm{nM}$ & $3.3 \mathrm{nM}^{\dagger}$ \\
hAChE-induced A $\beta(1-40)$ aggregation & $\mathrm{IC}_{50}=28.3 \pm 0.30 \mu \mathrm{M}$ & $\mathrm{IC}_{50}>>100 \mu \mathrm{M}^{\dagger}$ \\
$\mathrm{A} \beta(1-42)$ self-aggregation & $\mathrm{IC}_{50}=5.93 \pm 0.33 \mu \mathrm{M}$ & $\mathrm{IC}_{50}=60.3 \pm 11.2 \mu \mathrm{M}^{\ddagger}$ \\
BACE-1 & $\mathrm{IC}_{50}=108 \pm 23 \mathrm{nM}$ & $\mathrm{IC}_{50}=18 \pm 2 \mathrm{nM}^{\S}$
\end{tabular}

*For menadione.

${ }^{\dagger}$ For donepezil. ${ }^{44}$

${ }^{\ddagger}$ For tetracycline.

${ }^{\S}$ For statine-derivative.

accept electrons from NADH via NQO1, in comparison with the reference compound, menadione. The apparent $K_{\mathrm{m}}$ and $V_{\max }$ values of $12.7 \mu \mathrm{M}$ and $3480(\mu \mathrm{mol} / \mathrm{min}) /$ $\mathrm{mg}$, respectively, showed that $\mathbf{2}$ is a good substrate for NQO1, being not very different from menadione in terms of reduction by the enzyme. To further confirm the mechanism of action, we showed that both memoquin and menadione activities were sensitive to the NQO1 competitive inhibitor dicoumarol.

The cellular antioxidant activity of $\mathbf{2}$ against the formation of ROS was assessed in SH-SY5Y neuroblastoma cells after treatment with tert-butyl hydroperoxide. When treated with $\mathbf{2}$, the SH-SY5Y cells did not show a marked difference on ROS formation when compared to untreated cells $(75.47 \% \pm 8.00 \%$ and $100.00 \% \pm$ $6.00 \%$, respectively). Conversely, when the same experiment was repeated by pre-treating the cells with sulforaphane, a potent inducer of NQO1, ${ }^{42} 2$ produced a remarkable inhibitory effect on the ROS formation relative to the untreated cells $(56.60 \% \pm 4.00 \%$ and $103.77 \% \pm$ $11.00 \%$, respectively). This result clearly confirms that there is a direct relationship between the NQO1-mediated reduced form of $\mathbf{2}$ and its ability to prevent free radical formation and damage. Furthermore, a nonselective distribution of exogenous antioxidants usually accounts for their in vivo failure. In the case of $\mathbf{2}$, due to this sort of targeting, it might emerge in the "right" place and at the "right" time in vivo, overcoming the frequent miss between antioxidants and ROS. ${ }^{43}$

\section{Anti-AChE activity of memoquin}

The inhibitory potency of $\mathbf{2}$ against human AChE, expressed as $K_{\mathrm{i}}$ value, was equal to $2.60 \pm 0.48 \mathrm{nM}$. This is almost 10 times lower than that obtained in our system for the drug donepezil $\left(K_{\mathrm{i}}=20.5 \pm 3.3 \mathrm{nM}\right),{ }^{44}$ which is the most potent marketed AChEI. Kinetic measurements also revealed that the inhibition was of mixed type, showing a variation of both $V_{\max }$ and $K_{\mathrm{m}}$ in the presence of different concentrations of $\mathbf{2}$. We interpreted this result as evidence of the interaction of $\mathbf{2}$ with both the catalytic site and the PAS of the enzyme. Docking sim- ulations followed by molecular dynamics studies confirmed that $\mathbf{2}$ is able to simultaneously bind both the catalytic and peripheral sites of human AChE. ${ }^{33}$

\section{Anti-aggregating profile of memoquin}

AChE can act as a nucleating factor and promotes the conversion of soluble amyloid peptide into insoluble amyloid fibrils. ${ }^{45}$ It has also been demonstrated that AChEIs binding at the PAS of the enzyme can interfere with such a pro-aggregating action. ${ }^{22}$ When tested in a fluorimetric assay, ${ }^{44} \mathbf{2}$ showed a dose-dependent inhibitory effect on AChE-induced $\mathrm{A} \beta(1-40)$ aggregation $\left(\mathrm{IC}_{50}=28.3 \pm 0.3 \mu \mathrm{M}\right)$. Notably, of the classical AChEIs tested in the same conditions (tacrine, donepezil, galantamine, rivastigmine, propidium), only propidium, which is a specific inhibitor of PAS, displayed a comparable anti-aggregating potency, with an $\mathrm{IC}_{50}$ value of $12.6 \pm 0.3 \mu \mathrm{M} .{ }^{46}$ This result is in line with the numerous studies suggesting that modulation of the PAS may affect $\mathrm{A} \beta$ aggregation. ${ }^{46-51}$ It therefore seems likely that new AChEIs, which capitalize on different strengths, should be reconsidered as excellent candidates for future $\mathrm{AD}$ therapy. ${ }^{52}$

Given the recent evidence that $\mathrm{CoQ}$ inhibits $\mathrm{A} \beta$ aggregation $^{53}$ and that the p-benzoquinone moiety seems responsible for this activity, ${ }^{54}$ we could hypothesize similar anti-amyloidogenic and fibril-destabilizing effects for 2. Inhibition studies into self-aggregation of $A \beta(1-$ 42 ), which is the most amyloidogenic fragment found in the $\mathrm{AD}$ plaques, were also performed using a thioflavin T-based fluorometric method. ${ }^{44,55}$

When tested at concentrations ranging from 1.0 to 50 $\mu \mathrm{M}$, memoquin exhibited a strong dose-dependent inhibitory effect, with an $\mathrm{IC}_{50}$ value of $5.93 \pm 0.33 \mu \mathrm{M}$. When tested at an equimolar concentration $(50 \mu \mathrm{M})$ with $\mathrm{A} \beta(1-42)$, it was able to inhibit $95.5 \% \pm 0.35 \%$ of fibril formation, whereas, in the same experimental conditions, the AChEIs galantamine and tacrine did not show any significant inhibitory activity whatsoever. ${ }^{36}$ For comparison, in the same experimental conditions, the inhibitory 
activity of the quinone derivative tetracycline $e^{56}(50 \mu \mathrm{M}$; $\mathrm{A} \beta /$ tetr $1 / 1)$ was $44.95 \% \pm 12.06 \% .^{55}$

Consistent with the importance of benzoquinone as an anti-aggregating scaffold, we proved that $\mathbf{2}$ and other synthesized 2,5-diaminobenzoquinone analogues are inhibitors of $\mathrm{A} \beta$ aggregation. ${ }^{36}$ Furthermore, we have advanced the idea that the presence of a symmetrical protonated structure, with charges spaced across a hydrophobic scaffold by chains of a proper length and flexibility, is essential for activity. Despite these insights, the structureactivity relationships within the class deserve to be further explored. We hypothesize that the creation of more potent inhibitors will be facilitated by a better understanding of the modules to be appended to the central 2,5-diaminobenzoquinone core for an optimal activity. In turn, this might reveal the key residues of the amyloid surface that are critical for its aggregation and toxicity.

\section{Anti-BACE-1 activity of memoquin}

The ability of 2 to inhibit the amyloidogenic BACE-1 enzyme was also investigated. $A \beta$ is cleaved from the amyloid precursor protein (APP) by the combined actions of $\beta$-secretase (also called BACE-1- $\beta$-site APPcleaving enzyme), a membrane-bound aspartyl protease, and $\gamma$-secretase, a complex of several proteins, which cleaves APP within its transmembrane domain. ${ }^{57} \mathrm{Re}-$ markably, 2 was found to inhibit BACE-1 activity in a concentration-dependent manner at submicromolar concentrations. The $\mathrm{IC}_{50}$ value for memoquin inhibition of BACE-1 activity was $108.0 \pm 22.9 \mathrm{nM}$, whereas the well known statine-derived inhibitor (H-Lys-Thr-Glu-Glu-IleSer-Glu-Val-Asn-[Statine (3S,4S)]-Val-Ala-Glu-Phe-OH) in the same assay showed an $\mathrm{IC}_{50}$ value of $18 \pm 2 \mathrm{nM}$. This result is very promising for the possible therapeutic use of $2 .{ }^{58}$ Nevertheless, these data should be corroborated by cellular and molecular modeling studies.

\section{IN VIVO PROFILE}

The excellent multifunctional profile exhibited by memoquin in vitro motivated us to look for the in vivo proof of concept of our strategy. To test the action of 2 on AD neurodegeneration in vivo, we used different animal models. ${ }^{33,37}$ Here, we report on the data acquired on the AD11 mouse model, which exhibits a progressive neurodegenerative phenotype induced by expression of anti-NGF antibodies and which resembles many key features of human AD. Therefore, the AD11 mouse has been proposed as a comprehensive animal model for AD. ${ }^{59,60}$ In FIG. 4, we report a summary of all the in vivo activities of memoquin.

\section{Memoquin is able to restore cholinergic deficit}

AD11 mice are characterized by the presence of behavioral deficits, cholinergic dysfunction, deposition of endogenous $A \beta$, and tangle formation. ${ }^{61}$ To determine whether 2 can prevent the loss of choline acetyltransferase positive neurons in the central nervous system, it was first administered to AD11 mice at a very early stage of neurodegeneration (from 1.5 months to 2 months of age). The treatment was able to prevent cholinergic deficit in the basal forebrain, as well as the loss of cholinergic neurons of the basal nucleus of Myenert. More interestingly, the same results were obtained in mice aged 15 months, where the treatment caused an analogous improvement in the number of cholinergic neurons in the basal forebrain. ${ }^{37}$

\section{Memoquin reduces $\mathrm{A} \beta$ expression and accumulation}

In AD11 mice at 15 months of age, when neurodegeneration is full blown and an accumulation of extracellular $\mathrm{A} \beta$ deposits is evident, the oral administration of $\mathbf{2}$ caused a decrease in the number of $\mathrm{A} \beta$ plaques in comparison with placebo-treated mice. Although the number of plaques did not reach the level (equal to zero) shown by age-matched wild-type mice, the morphology of plaques remaining in memoquin-treated mice was different from that observed in untreated AD11 mice. ${ }^{37}$

\section{Memoquin reduces tau hyperphosphorylation}

In the somatodentritic compartments, AD11 mice showed an accumulation of intracellular tangles composed of the hyperphosphorylated form of the tau protein, which was not observed in the wild-type mice. At all ages, tau hyperphosphorylation was either completely (at 2 months of age) or partially (at 15 months of age) prevented by administration of $2{ }^{37}$

\section{Memoquin rescues behavioral deficits}

The efficacy of memoquin in rescuing behavioral deficits linked to attention and memory was assessed in AD11 mice after oral administration of $\mathbf{2}$ by means of the object recognition test (ORT). The same test was applied to nontransgenic mice treated with 220 minutes before the administration of scopolamine. After treatment with 2, neither AD 11 nor nontransgenic mice showed signs of amnesia. They were all able to distinguish familiar objects from new ones. ${ }^{37}$

\section{Comparison between memoquin's in vivo efficacy and that of galantamine and caproctamine}

In order to evaluate the relative contribution of the anti-AChE activity of memoquin with respect to its overall multiple profile, its efficacy in rescuing the AD phenotype in AD11 mice was compared to that of a benchmark AChEI (galantamine) and the diaminediamide derivative 1. In 6-month-old AD11 mice, galantamine was able to rescue the cholinergic deficit, the accumulation of $\mathrm{A} \beta$ in dystrophic neuritis, and the behavioral ORT deficit, but had no effect on the tau phenotype. Unexpectedly, caproctamine had no effect on the number 


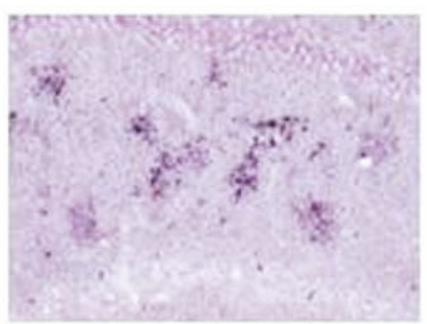

$A \beta$ plaque

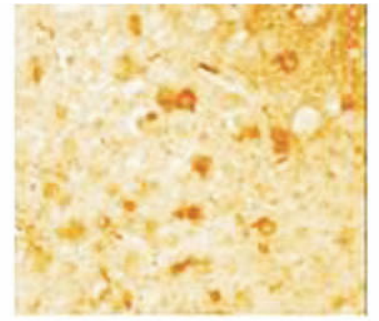

tau hyperphosphorylation

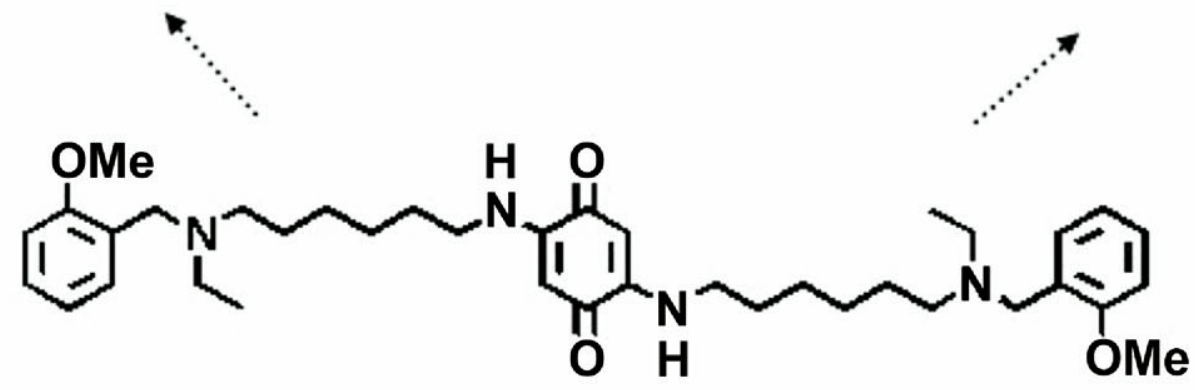

\section{Memoquin (2)}

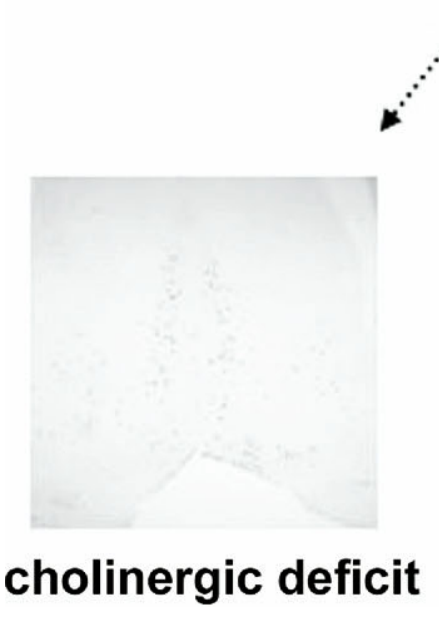

FIG. 4. Schematic representation of the in vivo MTDL profile of 2. of cholinergic neurons, nor on the $\mathrm{A} \beta$ deposition and tau hyperphosphorylation. Only $\mathbf{2}$ was able to affect the whole range of hallmarks that characterize AD-like neurodegeneration in AD11 mice. ${ }^{37}$ Thus, it was confirmed in preclinical disease models that a rationally designed MTDL has a better chance of affecting overall AD neurodegeneration by acting on multiple targets at different levels of the neurotoxic cascade. In particular, we can suggest that pharmacological activities other than the AChE inhibitory property contribute in a significant way to 2's remarkable effectiveness in the anti-NGF AD11 model.

\section{CONCLUSIONS}

Multiple, interrelated, biochemical pathways are thought to contribute to the neurodegenerative process of AD. In AD patients, multiple upstream factors are likely to impinge on the $\mathrm{AD}$ core, which is mainly represented by $\mathrm{A} \beta$ deposition, tau hyperphosphorylation, and cholinergic deficits.

Memoquin is a new drug candidate that was designed and synthesized in a deliberate attempt to find an MTDL able to interfere with different key target points of $\mathrm{AD}$ neurodegeneration. Taken together, the present results 
demonstrate that, in vivo, memoquin was able to ameliorate the cholinergic and behavioral impairment, the expression and deposition of $\mathrm{A} \beta$, and the hyperphosphorylation and deposition of tau at different stages of neurodegeneration-even in aged mice. Furthermore, in the AD11 model, memoquin outperformed benchmark AChEI anti-Alzheimer drugs (i.e., galantamine). In addition, memoquin showed other promising properties: a good oral bioavailability, blood-brain barrier penetration, and a favorable safety profile in preclinical non-regulatory acute and chronic toxicology studies-being well tolerated after prolonged administration. ${ }^{62}$ Regarding the possible concerns about quinone toxicity, it should be mentioned that safe and modern quinone-containing drugs already exist, and have even been used for children and infants. ${ }^{63}$

The 1,4-benzoquinone moiety, typical of memoquin, $\mathrm{CoQ}$, and its synthetic analogue idebenone, is known to act as a radical scavenger ${ }^{38}$ and to protect against the oxidative stress induced by $\mathrm{A} \beta$ accumulation. In vitro, memoquin showed antioxidant capacity. In addition, the NQO1 enzyme, whose activity is increased in hippocampal pyramidal neurons of patients with $\mathrm{AD},{ }^{41,64}$ might convert memoquin into the hydroquinone form, which can function as a potent and targeted antioxidant. The important goal of locating the scavenger in sufficient proximity to its targets (ROS), so that these can be reached without the spontaneous decay or enzymatic neutralization of the messenger, seems to be fulfilled. ${ }^{65}$

Memoquin was also able to inhibit AChE catalytic action and both $\mathrm{AChE}$ - and self-induced $\mathrm{A} \beta$ aggregation. Moreover, it seemed to be a good non-peptidic BACE-1 inhibitor, with the potential to inhibit the actual formation of $\mathrm{A} \beta$ peptide.

When compared to a marketed AChEI (such as galantamine), the overall profile of memoquin is indeed due to its multi-targeted activity, and not exclusively to its ability to inhibit the AChE enzyme. In particular, for example, the remarkable ability of memoquin to rescue the tau-related neurofibrillary degeneration further indicates the important role of the multiple mechanisms of action, with a suggested neuroprotective activity linked to its antioxidant properties.

Therefore, memoquin emerges as a true disease-modifying agent in contrast to the presently marketed drugs, and a comprehensive biological and pharmacological profile of the molecule has been reported in the patent filed by the University of Bologna. ${ }^{37}$

In light of the inappropriateness of the currently available drugs to confront the multi-faceted etiology of AD, the MTDL approach stands as a new and promising way of discovering small molecules able to address the biological complexity underlying neurodegenerative pathways.

In summary, the discovery of compounds with an MTDL profile as described in this report suggests that future efforts in this field may ultimately lead to a new therapy for AD. We are confident that MTDLs will be helpful tools in the challenge to understand, treat, and defeat AD.

\section{Disclosure}

The authors state no conflict of interest.

Acknowledgments: This work was supported by a grant from MIUR (FIRB RBNE03FH5Y) and the University of Bologna.

\section{REFERENCES}

1. Brookmeyer RS, Johnson E, Ziegler-Graham K, Arrighi HM. Forecasting the global burden of Alzheimer's disease. Alzheimer Dementia 2007;3:186-191.

2. Ferri CP, Prince M, Brayne C, Brodaty H, Fratiglioni L, Ganguli $\mathrm{M}$, et al. Global prevalence of dementia: a Delphi consensus study. Lancet 2005;366:2112-2117.

3. Small DH. Acetylcholinesterase inhibitors for the treatment of dementia in Alzheimer's disease: do we need new inhibitors? Expert Opin Emerg Drugs 2005;10:817-825.

4. Roberson ED, Mucke L. 100 years and counting: prospects for defeating Alzheimer's disease. Science 2006;314:781-784.

5. Hardy J, Selkoe DJ. The amyloid hypothesis of Alzheimer's disease: progress and problems on the road to therapeutics. Science 2002;297:353-356.

6. Golde TE. Disease modifying therapy for AD? J Neurochem 2006 ; 99:689-707.

7. Small DH, Mok SS, Bornstein JC. Alzheimer's disease and Abeta toxicity: from top to bottom. Nat Rev Neurosci 2001;2:595-598.

8. Frantz S. Drug discovery: playing dirty. Nature 2005;437:942943.

9. Iqbal K, Grundke-Iqbal I. Alzheimer disease is multifactorial and heterogeneous. Neurobiol Aging 2000;21:901-902; discussion 903-904.

10. Morphy R, Rankovic Z. Designed multiple ligands. An emerging drug discovery paradigm. J Med Chem 2005;48:6523-6543.

11. Farlow MR. Utilizing combination therapy in the treatment of Alzheimer's disease. Expert Rev Neurother 2004;4:799-808.

12. Farlow MR, Miller ML, Pejovic V. Treatment options in Alzheimer's disease: maximizing benefit, managing expectations. Dement Geriatr Cogn Disord 2008;25:408-422.

13. Toews ML, Bylund DB. Pharmacologic principles for combination therapy. Proc Am Thorac Soc 2005;2:282-289; discussion 290291.

14. Cavalli A, Bolognesi ML, Minarini A, Rosini M, Tumiatti V, Recanatini M, et al. Multi-target-directed ligands to combat neurodegenerative diseases. J Med Chem 2008;51:347-372.

15. Small G, Dubois B. A review of compliance to treatment in Alzheimer's disease: potential benefits of a transdermal patch. Curr Med Res Opin 2007;23:2705-2713.

16. Van der Schyf CJ, Mandel S, Geldenhuys WJ, Amit T, Avramovich Y, Zheng H, et al. Novel multifunctional anti-Alzheimer drugs with various CNS neurotransmitter targets and neuroprotective moieties. Curr Alzheimer Res 2007;4:522-536.

17. Melchiorre C, Andrisano V, Bolognesi ML, Budriesi R, Cavalli A, Cavrini V, et al. Acetylcholinesterase noncovalent inhibitors based on a polyamine backbone for potential use against Alzheimer's disease. J Med Chem 1998;41:4186-4189.

18. Doods HN, Quirion R, Mihm G, Engel W, Rudolf K, Entzeroth M, et al. Therapeutic potential of CNS-active M2 antagonists: novel structures and pharmacology. Life Sci 1993;52:497-503.

19. Munoz-Torrero D, Camps P. Dimeric and hybrid anti-Alzheimer drug candidates. Curr Med Chem 2006;13:399-422.

20. Alvarez A, Alarcon R, Opazo C, Campos EO, Munoz FJ, Calderon $\mathrm{FH}$, et al. Stable complexes involving acetylcholinesterase and amyloid-beta peptide change the biochemical properties of the enzyme and increase the neurotoxicity of Alzheimer's fibrils. J Neurosci 1998;18:3213-3223. 
21. Campos EO, Alvarez A, Inestrosa NC. Brain acetylcholinesterase promotes amyloid-beta-peptide aggregation but does not hydrolyze amyloid precursor protein peptides. Neurochem Res 1998;23:135140 .

22. Inestrosa NC, Alvarez A, Perez CA, Moreno RD, Vicente M, Linker C, et al. Acetylcholinesterase accelerates assembly of amyloid-beta-peptides into Alzheimer's fibrils: possible role of the peripheral site of the enzyme. Neuron 1996;16:881-891.

23. Bourne Y, Taylor P, Bougis PE, Marchot P. Crystal structure of mouse acetylcholinesterase. A peripheral site-occluding loop in a tetrameric assembly. J Biol Chem 1999;274:2963-2970.

24. Castro A, Martinez A. Peripheral and dual binding site acetylcholinesterase inhibitors: implications in treatment of Alzheimer's disease. Mini Rev Med Chem 2001;1:267-272.

25. Recanatini M, Valenti P. Acetylcholinesterase inhibitors as a starting point towards improved Alzheimer's disease therapeutics. Curr Pharm Des 2004;10:3157-3166.

26. Castro A, Martinez A. Targeting Beta-amyloid pathogenesis through acetylcholinesterase inhibitors. Curr Pharm Des 2006;12: 4377-4387.

27. Melchiorre C, Antonello A, Banzi R, Bolognesi ML, Minarini A, Rosini M, et al. Polymethylene tetraamine backbone as template for the development of biologically active polyamines. Med Res Rev 2003;23:200-233.

28. Bolognesi ML, Minarini A, Rosini M, Tumiatti V, Melchiorre C. From dual binding site acetylcholinesterase inhibitors to multitarget-directed ligands (MTDLs): a step forward in the treatment of Alzheimer's disease. Mini Rev Med Chem 2008;8:960-967.

29. Gutzmann H, Hadler D. Sustained efficacy and safety of idebenone in the treatment of Alzheimer's disease: update on a 2-year doubleblind multicentre study. J Neural Transm Suppl 1998;54:301-310.

30. Hirai K, Hayako H, Kato K, Miyamoto M. Idebenone protects hippocampal neurons against amyloid beta-peptide-induced neurotoxicity in rat primary cultures. Naunyn Schmiedebergs Arch Pharmacol 1998:358:582-585.

31. Moreira PI, Santos MS, Oliveira CR. Alzheimer's disease: a lesson from mitochondrial dysfunction. Antioxid Redox Signal 2007;9: $1621-1630$.

32. Adkins JC, Noble S. Idebenone: a review of its use in mild to moderate Alzheimer's disease. CNS Drugs 1998;9:403-419.

33. Cavalli A, Bolognesi ML, Capsoni S, Andrisano V, Bartolini M, Margotti E, et al. A small molecule targeting the multifactorial nature of Alzheimer's disease. Angew Chem Int Ed Engl 2007;46: 3689-3692.

34. Nesterov EE, Skoch J, Hyman BT, Klunk WE, Bacskai BJ, Swager TM. In vivo optical imaging of amyloid aggregates in brain: design of fluorescent markers. Angew Chem Int Ed Engl 2005;44:54525456.

35. Bolognesi ML, Banzi R, Minarini A, Melchiorre C, inventors. Process for preparation of 1,4-benzoquinone-2,5-diamines by reaction of amines with 1,4-benzoquinones bearing leaving groups at the 2- and 5-positions. PCT Int Appl WO 2006134457.

36. Bolognesi ML, Banzi R, Bartolini M, Cavalli A, Tarozzi A, Andrisano $\mathrm{V}$, et al. Novel class of quinone-bearing polyamines as multi-target-directed ligands to combat Alzheimer's disease. J Med Chem 2007;50:4882-4897.

37. Andrisano V, Bartolini M, Bolognesi ML, Cavalli A, Melchiorre C, Recanatini M, inventors. Preparation of 2,5-bis-diamine$[1,4]$ benzoquinone derivatives for the treatment of Alzheimer's disease and a process for their preparation and intermediates therefor. PCT Int Appl WO 2003087035.

38. Mordente A, Martorana GE, Minotti G, Giardina B. Antioxidant properties of 2,3-dimethoxy-5-methyl-6-(10-hydroxydecyl)-1,4benzoquinone (idebenone). Chem Res Toxicol 1998;11:54-63.

39. Raina AK, Templeton DJ, Deak JC, Perry G, Smith MA. Quinone reductase (NQO1), a sensitive redox indicator, is increased in Alzheimer's disease. Redox Rep 1999;4:23-27.

40. Moreira PI, Siedlak SL, Aliev G, Zhu X, Cash AD, Smith MA, et al. Oxidative stress mechanisms and potential therapeutics in Alzheimer disease. J Neural Transm 2005;112:921-932.

41. SantaCruz KS, Yazlovitskaya E, Collins J, Johnson J, DeCarli C. Regional NAD(P)H:quinone oxidoreductase activity in Alzheimer's disease. Neurobiol Aging 2004;25:63-69.
42. Kang YH, Pezzuto JM. Induction of quinone reductase as a primary screen for natural product anticarcinogens. Methods Enzymol 2004;382:380-414.

43. Shen L, Ji HF, Zhang HY. How to understand the dichotomy of antioxidants. Biochem Biophys Res Commun 2007;362:543-545.

44. Bartolini M, Bertucci C, Cavrini V, Andrisano V. Beta-Amyloid aggregation induced by human acetylcholinesterase: inhibition studies. Biochem Pharmacol 2003;65:407-416.

45. Alvarez A, Opazo C, Alarcon R, Garrido J, Inestrosa NC. Acetylcholinesterase promotes the aggregation of amyloid-beta-peptide fragments by forming a complex with the growing fibrils. J Mol Biol 1997;272:348-361.

46. Bolognesi ML, Andrisano V, Bartolini M, Banzi R, Melchiorre C. Propidium-based polyamine ligands as potent inhibitors of acetylcholinesterase and acetylcholinesterase-induced amyloid-beta aggregation. J Med Chem 2005;48:24-27.

47. Piazzi L, Rampa A, Bisi A, Gobbi S, Belluti F, Cavalli A, et al. 3-(4-[[Benzyl(methyl)amino]methyl]phenyl)-6,7-dimethoxy2H-2-chromenone (AP2238) inhibits both acetylcholinesterase and acetylcholinesterase-induced beta-amyloid aggregation: a dual function lead for Alzheimer's disease therapy. J Med Chem 2003;46:2279-2282.

48. Dickerson TJ, Beuscher AE 4th, Rogers CJ, Hixon MS, Yamamoto $\mathrm{N}, \mathrm{Xu} \mathrm{Y}$, et al. Discovery of acetylcholinesterase peripheral anionic site ligands through computational refinement of a directed library. Biochemistry 2005;44:14845-14853.

49. Munoz-Ruiz P, Rubio L, Garcia-Palomero E, Dorronsoro I, del Monte-Millan M, Valenzuela R, et al. Design, synthesis, and biological evaluation of dual binding site acetylcholinesterase inhibitors: new disease-modifying agents for Alzheimer's disease. J Med Chem 2005;48:7223-7233.

50. Xie Q, Wang H, Xia Z, Lu M, Zhang W, Wang X, et al. Bis-(-)nor-meptazinols as novel nanomolar cholinesterase inhibitors with high inhibitory potency on amyloid-beta aggregation. J Med Chem 2008;51:2027-2036.

51. Camps P, Formosa X, Galdeano C, Gomez T, Munoz-Torrero D, Scarpellini M, et al. Novel donepezil-based inhibitors of acetyland butyrylcholinesterase and acetylcholinesterase-induced betaamyloid aggregation. J Med Chem 2008;51:3588-3598.

52. Rees TM, Brimijoin S. The role of acetylcholinesterase in the pathogenesis of Alzheimer's disease. Drugs Today (Barc) 2003; 39:75-83.

53. Ono K, Hasegawa K, Naiki H, Yamada M. Preformed beta-amyloid fibrils are destabilized by coenzyme Q10 in vitro. Biochem Biophys Res Commun 2005;330:111-116.

54. Tomiyama T, Shoji A, Kataoka K, Suwa Y, Asano S, Kaneko H, et al. Inhibition of amyloid beta protein aggregation and neurotoxicity by rifampicin. Its possible function as a hydroxyl radical scavenger. J Biol Chem 1996;271:6839-6844.

55. Bartolini M, Bertucci C, Bolognesi ML, Cavalli A, Melchiorre C, Andrisano V. Insight into the kinetic of amyloid beta (1-42) peptide self-aggregation: elucidation of inhibitors' mechanism of action. Chembiochem 2007;8:2152-2161.

56. Forloni G, Colombo L, Girola L, Tagliavini F, Salmona M. Antiamyloidogenic activity of tetracyclines: studies in vitro. FEBS Lett 2001;487:404-407.

57. Dewachter I, Van Leuven F. Secretases as targets for the treatment of Alzheimer's disease: the prospects. Lancet Neurol 2002;1:409416.

58. Melnikova I. Therapies for Alzheimer's disease. Nat Rev Drug Discov 2007;6:341-342.

59. Capsoni S, Ugolini G, Comparini A, Ruberti F, Berardi N, Cattaneo A. Alzheimer-like neurodegeneration in aged antinerve growth factor transgenic mice. Proc Natl Acad Sci U S A 2000;97:68266831.

60. Capsoni S, Cattaneo A. On the molecular basis linking Nerve Growth Factor (NGF) to Alzheimer's disease. Cell Mol Neurobiol 2006;26:619-633.

61. Capsoni S, Giannotta S, Cattaneo A. Beta-amyloid plaques in a model for sporadic Alzheimer's disease based on transgenic antinerve growth factor antibodies. Mol Cell Neurosci 2002;21:15-28.

62. Capsoni S, Andrisano V, Bartolini M, Bolognesi ML, Cavalli A, Margotti E, et al. S4-04-04 Memoquin, a novel multifunctional 
compound for Alzheimer's disease: An up-date on preclinical studies. Alzheimer Dementia 2006;2(3 Suppl 1):S73-S74.

63. Liu K, Xu L, Szalkowski D, Li Z, Ding V, Kwei G, et al. Discovery of a potent, highly selective, and orally efficacious smallmolecule activator of the insulin receptor. J Med Chem 2000;43: $3487-3494$
64. Wang Y, Santa-Cruz K, DeCarli C, Johnson JA. NAD(P)H:quinone oxidoreductase activity is increased in hippocampal pyramidal neurons of patients with Alzheimer's disease. Neurobiol Aging 2000;21:525-531.

65. Azzi A. Oxidative stress: A dead end or a laboratory hypothesis? Biochem Biophys Res Commun 2007;362:230-232. 